\title{
Do woodsticks work?
}

\author{
Abstracted from \\ Hoenderdos NL, Slot DE, Paraskevas S, Van der Weijden GA. \\ The efficacy of woodsticks on plaque and gingival inflammation: a systematic review. \\ Int J Dent Hyg 2008; 6: 280-289 \\ Address for correspondence: NL Hoenderdos, Academic Centre for Dentistry Amsterdam, \\ Department of Periodontology, Louwesweg 1, 1066 EA Amsterdam, The Netherlands. \\ E-mail:n.hoenderdos@acta.nl
}

\section{Question: Is using woodsticks as an adjunct to toothbrushing more effective at reducing periodontal inflammation than toothbrushing alone?}

Data Sources Medline and the Cochrane Central Register of Controlled Trials were used to find relevant research.

Study selection Studies were screened independently by two reviewers. Randomised controlled trials and controlled clinical trials were selected if they were conducted in individuals of over 18 years of age who were in good general health, and which used plaque, bleeding, gingivitis or pocket depth as outcome measures. Case reports, letters, and narrative or historical reviews were excluded and only English-language papers were considered.

Data extraction and synthesis Because of the heterogeneity of the studies' designs, a qualitative summary was presented.

Results Seven publications describing eight clinical experiments met the inclusion criteria. The improvement in gingival health observed in the studies represented a significant incremental benefit realised by the use of triangular woodsticks. None of the studies that scored plaque demonstrated any significant advantage of the use of woodsticks over alternative methods of plaque removal in people who had gingivitis. Conclusions The evidence from clinical controlled trials shows that woodsticks do not have an additional effect on visible interdental plaque, but do, however, help improve interdental gingival inflammation.

\section{Commentary}

Plaque accumulation on initially healthy gingivae has been associated with induction of gingival inflammation. Subsequent removal of this plaque has been shown to restore gingival health. ${ }^{1}$ This review considers the efficacy of woodsticks on the removal of plaque and the effect on gingival inflammation.

The literature search here was confined to studies in the English language. This may have limited the authors' access to data from possible articles in other languages, to which the Cochrane Central Register of Controlled Trials usually allows access, and indeed provides translations. The review was conducted according to standard protocols for systematic reviews.

Data analysis revealed considerable heterogeneity in many aspects of the included studies, so a meta-analysis could not be carried out. Out of just seven studies included for data abstraction, two were not randomised. Four were of crossover design and four of parallel arm (one study had both). The study sizes ranged from 10 to 161 subjects, and control groups were varied. The indices used for plaque and bleeding also varied. Some of the plaque indices require subjective interpretation, which can increase bias. It appeared that some studies did not provide instruction on plaque control; also, some had professional intervention and others did not. Three studies reported bleeding scores that suggested a benefit from use of woodsticks; only two studies reported gingival indices, which showed no difference between woodstick and control groups.

With so many variables contributing to plaque removal, to gingival inflammation and to the assessment of these indices, standardised study design is difficult. One of the findings of this review was that woodsticks did not make any difference to interproximal plaque removal, but that their use did influence (reduce) gingival inflammation. With the lack of correlation between the collected data, it is clear that more studies with larger numbers of subjects and study designs that follow CONSORT (www.consort-statement.org) guidelines are needed to clarify the efficacy of woodsticks on plaque reduction and their affect on gingival inflammation. With the limited data within this review, it is difficult to draw any meaningful conclusions on the efficacy of woodsticks.

Richard Tucker

Unit of Periodontology, University College London Eastman Dental Institute, London, UK

1. Löe $H$, Theilade E, Jensen SB. Experimental gingivitis in man. J Periodontol 1965; 36: 177-187.

Evidence-Based Dentistry (2009) 10, 43. doi:10.1038/sj.ebd.6400646 